Revue d'histoire de l'Amérique française

RAS REVUE D.HISTOIRE DE L'AMÉRIQUE FRANÇAISE

\title{
Les derniers jours de Maisonneuve et Philippe de Turmenyes
}

\section{Robert Le Blant}

Volume 13, numéro 2, septembre 1959

URI : https://id.erudit.org/iderudit/301970ar

DOI : https://doi.org/10.7202/301970ar

Aller au sommaire du numéro

Éditeur(s)

Institut d'histoire de l'Amérique française

ISSN

0035-2357 (imprimé)

1492-1383 (numérique)

Découvrir la revue

Citer ce document

Le Blant, R. (1959). Les derniers jours de Maisonneuve et Philippe de Turmenyes. Revue d'histoire de l'Amérique française, 13(2), 262-280.

https://doi.org/10.7202/301970ar d'utilisation que vous pouvez consulter en ligne.

https://apropos.erudit.org/fr/usagers/politique-dutilisation/ 


\section{DOCUMENTS INÉDITS}

\section{LES DERNIERS JOURS DE MAISONNEUVE ET PHILIPPE DE TURMENYES 14 avril 1666 - 9 septembre 1676 - 3 août 1699}

Paul de Chomedey habitait à Paris, rue Saint-Martin, paroisse Saint-Médéric, le 14 avril 1666, date à laquelle il donna devant le notaire Bernard Mousnier ${ }^{1}$ une procuration concernant une rente sur la ville de Paris et le 29 octobre 1667, lorsqu'avec Charles de Lauzon, prêtre official de Monseigneur de Laval, évêque de Pétrée, il signa devant le notaire Auvray, ${ }^{2}$ à la suite d'une procuration donnée par Jeanne Mance le 2 août précédent, devant Romain Becquet, notaire à Québec, certifiée par Talon et Patoulet.

Jeanne Mance constituait comme procureur général pour administrer et régler toutes ses affaires en France noble et discrète personne Nicolas d'Ollebeau, prêtre chanoine de la SainteChapelle à Paris. Elle déclarait être héritière pour moitié de demoiselle Anne Rober, dite Mance, sa cousine germaine, demeurant à Beaumont-sur-Oise près Paris ${ }^{3}$. Sa signature était renforcée par celles de Louis Fin et Charles Pingard, habitants de Québec.

Maisonneuve bénéficia par acte du 4 avril 1668 passé devant Le Vasseur le jeune, notaire au Châtelet, de la constitution d'une rente annuelle de 500 livres effectuée par Alexandre Le Ragois, de Bretonvilliers, supérieur de Saint-Sulpice et messire Antoine

${ }^{1} \mathrm{Cf}$. Minutier Central, étude 112, 114, minutes de Bernard Mousnier, aux Archives Nationales.

2 Cf. Idem, étude 9, no 434.

3 Canton de l'Isle-Adam, arrondissement de Pontoise, département de Seine et Oise. 
Raguier de Pourché, curé de Saint-Sulpice, agissant pour le Séminaire du même nom ${ }^{4}$.

La perte de l'acte, qu'il consentit ${ }^{5}$ le 3 juillet 1668 devant le notaire Jean-Baptiste Bizet ${ }^{6}$, empêche de connaître le lieu de la résidence de Paul de Chomedey à cette date. Il s'agissait d'un engagement de payer 950 livres à Philippe de Turmenyes qui semble avoir été son meilleur ami à la fin de son existence. Né vers 1630 , ce personnage appartenait à une famille comptant de nombreux représentants à Beaumont-sur-Oise. Petit-fils de Me Jean de Turmenyes, procureur en cette localité et de Jacqueline de La Brosse à la succession de laquelle il renonça le 2 mai 1679 suivant acte passé devant Mousnier ${ }^{7}$, il était le frère de François et Nicolas de Turmenyes, de Barbe, femme de Louis Le Gaast, sieur de Beaumont, d'Anne, épouse de Louis Maillard, de Marie de Turmenyes, femme de Nicolas de Fourcroy ${ }^{8}$ et probablement le neveu de Marguerite de Turmenyes. Celle-ci était certainement la tante de Nicolas de Turmenyes, sieur de Belle-Isle, de Jean de Turmenyes, seigneur d'Angerenne et de Marthe de Turmenyes qui étaient ses héritiers chacun pour un tiers, aux termes d'un acte passé le 22 novembre 1670 devant Rissen et Rozé, notaires à Beaumont sur Oise, par lequel ils se désistèrent au profit d'Étienne et Charles de Turmenyes de leurs oppositions sur les biens de la succession de François de Turmenyes ${ }^{9}$.

Oncle d'Anselme de Maillard, prêtre ${ }^{10}$, Philippe, qui nous intéresse, vit l'orthographe de son nom de famille connaitre des vicissitudes pour la solution desquelles nous avons suivi sa pro-

4 Cf. Substitution du 6 septembre 1675 passée devant Auvray, étude 9, no 451. Nous n'avons pas retrouvé l'acte du 4 avril 1668 dont l'original ou tout au moins une expédition se trouverait à la Bibliothèque municipale de Montréal d'après l'inventaire provisoire des Archives d'Ottawa, Fonds des Manuscrits, no 18, (1954), 18 (3). Les actes de tout le mois d'avril 1688 manquent aux minutes de Claude Le Vasseur, étude 98, no 227.

5 Cf. Inventaire après décès de Paul de Chomedey. Minutes de Bernard Mousnier, étude 112, no 164.

6 Etude 21, répertoire.

7 Cf. Etude 112, no 175 .

8 Cf. Dossiers Bleus 650, art. Turmenyes, à la Bib. Nat.

$9 \mathrm{Cf}$. Minutes de Bernard Mousnier, étude 112, no 134.

$10 \mathrm{Cf}$. Acte de Mousnier du 27 juillet 1679, déjâ cité. 
pre signature. Il exerça des activités diverses, étant intitulé, dans les actes bourgeois de Paris, écuyer, conseiller du roi, contrôleur ordinaire des guerres au régiment des gardes suisses et administrateur de l'hôpital Sainte-Reine en Bourgogne ${ }^{11}$.

Les difficultés financières de Maisonneuve étaient excusables, car d'après un certificat de Peuvret, greffier au Conseil souverain de Québec, du 16 octobre 1668, il lui était encore dû 4000 livres sur ses appointements de gouverneur de Montréal ${ }^{12}$. L'amitié n'était pas exclusive des procès à l'époque, car Turmenyes obtint une sentence de condamnation pour 1000 livres le 8 mars 1669 et Maisonneuve s'acquitta le $14^{13}$ en aliénant le même jour, moyennant 1000 livres au profit de demoiselle Gabrielle Le Messier, veuve de noble homme Bertrand Hardouin de Saint-Jacques, docteur en médecine de la Faculté de Paris, 50 livres de rente gagées sur le capital de 10800 livres à lui dû par la compagnie des marchands tanneurs de Troyes ${ }^{14}$ en contrepartie d'une rente de 600 livres consentie par acte passé devant Thévignon, notaire de Troyes, le $1^{\text {er }}$ août $1652^{15}$. La dame de Saint-Jacques était une ancienne connaissance qui lui avait déjà fourni 3000 livres dans des conditions analogues suivant acte passé devant André Bouret, notaire au Châtelet de Paris, le 16 mars 1657. Maisonneuve demeurait alors chez Pierre Blondel, bourgeois de Paris, qui remboursa la somme le 29 mars 1663, en vertu d'une procuration donnée le 12 avril 1657 devant le notaire de Saint-Waast ${ }^{16}$. Pierre Blondel, bien connu des historiens de la Société de Notre-Dame-de-Montréal, était marié avec Marie Blaté et habitait rue aux Ours, paroisse Saint-Leu-et-Saint-Gilles, le 21 novembre 1648, lorsque sa fille Denise fut admise au monastère de Saint-Augustin à Picpus, suivant acte passé devant Renault Vaultier, notaire au Châtelet ${ }^{17}$.

11 Cf. Minutes d'Auvray, étude 9, no 451.

$12 \mathrm{Cf}$. Inventaire après décès, déjà cité.

13 Cf. Idem.

14 Cf. Minutes d'Auvray, déjà citées, no 437.

$15 \mathrm{Cf}$. Inventaire après décès, déjà cité.

16 Cf. Etude 99, no 195. La quittance est en marge de l'acte et la procuration manque aux minutes de Charles-François de Saint-Waast, étude 73 , no 432.

17 Cf. Y 187, fol. 37, aux Archives Nationales, d'après étude 112, no 52. 
Le paladin de la Nouvelle-France habitait, le 8 mars 1669, dans une petite maison nouvellement construite dans les dépendances du couvent des révérends pères de la Doctrine Chrétienne, sur le fossé, entre les portes Saint-Marcel et Saint-Victor, où, pour deux pièces situées l'une au-dessus de l'autre, il paya un loyer paraissant considérable de 27 écus par an, soit 81 livres et environ 162,000 fcs français de $1958 .{ }^{18}$

Chomedey attesta devant le notaire Bernard Mousnier, ${ }^{19}$ le 22 novembre 1670, que Marguerite Bourgeois était arrivée en France au cours du mois d'octobre précédent pour augmenter le nombre des filles de sa communauté et obtenir des lettres patentes du roi en faveur de son établissement, déjà muni de certificats délivrés par Mgr de Laval, Rémy de Courcelles et Talon. Les lettres datées à Dunkerque en mai 1671, vérifiées au parlement de Paris, le 20 juin suivant, portèrent confirmation de l'établissement d'une communauté de filles sous le nom de Congrégation de Notre-Dame, dans l'île de Montréal, pour l'instruction des jeunes filles. Maisonneuve devait se sentir bien oublié, car il fit annexer à son attestation la lettre originale du dernier avril 1653 signée de Louis XIV ${ }^{20}$ lui ordonnant de conduire le secours d'hommes pour la défense de l'île de Montréal sur les vaisseaux qu'il trouverait les plus commodes. L'attestation proprement dite comprenait un court historique des exploits de Marguerite Bourgeois dont le gouverneur avait fait la connaissance seulement à Nantes en 1653 et dont l'activité avait comporté l'avance des fonds nécessaires pour une exploitation agricole avec bestiaux et la construction de deux corps de logis destinés aux filles.

Marguerite Bourgeois resta en France au moins jusqu'au 26 juin 1671, date à laquelle, considérée comme logeant sur le fossé entre les portes de St-Victor et de St-Marcel, c'est-à-dire vraisemblablement chez Maisonneuve, elle gratifia ce dernier, suivant acte passé devant le notaire Auvray en la maison et bureau de l'hôpital de Sainte-Reine, rue Saint-Martin, d'une procuration fort complète et détaillée aux fins d'agir partout où besoin serait

$18 \mathrm{Cf}$. Inventaire après décès, déjà cité.

19 Cf. Etude 112, no 134.

20 Cf. Idem. 
pour les affaires de sa congrégation. ${ }^{21}$ Le gouverneur reconnut devoir 264 livres à Louis Fin suivant acte passé devant Auvray le 10 mai $1673^{22}$ et c'est seulement le 29 mai 1675 que Paul de Chomedey établit, lui aussi, une procuration, mais celle-là en blanc, pour recouvrer les 4000 livres d'appointements, près de 8 millions de franes de nos jours à lui dues au moins depuis le 16 octobre 1668 . Il rédigea en même temps un récit des démarches effectuées pour tenter ce recouvrement dont on trouve seulement la mention dans l'inventaire fait après sa mort.

Suivant acte du 6 septembre suivant passé devant le notaire Auvray, Maisonneuve se substitua Philippe de Turmenyes ${ }^{23}$ pour l'exécution de la procuration donnée par Marguerite Bourgeois. Il hui donna en même temps la jouissance viagère comprenant 1450 livres d'arrérages impayés des 500 livres de la rente constituée pour le compte du séminaire de Saint-Sulpice. Turmenyes en donna quittance, suivant acte passé devant le notaire Le Vasseur, le 6 avril $1676 .^{24}$

Malade et alité chez lui, dans la salle du premier étage qualifiée entresol donnant sur la cour des révérends pères de la Doctrine Chrétienne et sur le fossé, Maisonneuve dicta, le 8 septembre 1676, aux notaires parisiens Torinon et Aumont un testament qui resta en la possession de ce dernier."5 Se contentant, pour recommander son âme à Dieu, de recourir à l'intervention de la Sainte Vierge et des Saints, sans implorer le secours des anges ainsi que le firent d'autres associés de Notre-Dame-deMontréal, ${ }^{26}$ il ordonna l'inhumation de son corps dans l'église des révérends pères de la Doctrine Chrétienne de la maison de SaintCharles auxquels il légua 500 livres pour le droit de sépulture et les prières à son intention, s'en rapportant à son exécuteur tes-

21 Cf. Etude 9, no 442.

$22 \mathrm{Cf}$. Inventaire après décès, déjà cité. L'acte manque aux minutes d'Auvray, étude 9, no 446.

23 Cf. Etude 9, no 451.

$24 \mathrm{Cf}$. Inventaire après décès, déjà cité. L'acte manque aux minutes de Claude Le Vasseur, étude 98, no 257.

$25 \mathrm{Cf}$. Minutes d'Adrien Aumont, étude 17, no 364.

26 Cf. Testaments de Bertrand Drouart et d'Alexandre Le Ragois de Bretonviliers, minutes de Muret, étude 91 , no 349 et minutes de Claude Le Vasseur, étude 98, no 257. 
tamentaire, Philippe de Turmenyes, pour le surplus de ses obsèques, des services et d'autres prières.

Le testateur constitua une bonne distribution de ce qu'il possédait, léguant 2000 livres à la Congrégation de Notre-Damede Montréal dont Marguerite Bourgeois était supérieur, 300 livres en plus de ce qu'il lui devait à Louis Fin, son serviteur, 1000 livres aux Hospitalières de Saint-Joseph de Villemarie, 1000 livres aux Filles de la Congrégation de Troyes dont faisaient partie sa sœur et sa nièce, 300 livres à Monsieur Le Bey, promoteur de l'évêque de Troyes, 400 livres aux pauvres de l'hôpital SainteReine.

Il reconnut devoir 400 livres à Jacques Le Bert et Charles Le Moyne, marchands au Canada, 100 livres constituant le capital d'une rente à Gabrielle Le Messier, stipulant que ces legs et dettes ainsi énoncés devaient être acquittés sur les 10800 livres de rente au denier dix-huit et les arrérages dus par la communauté des tanneurs de Troyes, ainsi qu'un legs supplémentaire de 200 livres au profit de Robert Carron, maitre joueur de luth.

Philippe de Turmenyes reçut tous les meubles, habits et linges qui pourraient exister au moment du décès, à l'exception de quelques chemises et habits pouvant servir à Louis Fin à qui l'exécuteur testamentaire était prié de les laisser. Turmenyes se vit aussi gratifier de la moitié de ce qui pourrait rester disponible sur la créance contre les tanneurs de Troyes, l'autre moitié devant être remise au révérend Père Charles, provincial des révérends pères de la Doctrine Chrétienne, pour en disposer pieusement ainsi qu'il l'entendrait.

La nièce héritière, Marie Bouvot, paraissait, cependant, pouvoir retirer quelque chose de la succession comprenant une somme de 160 livres en espèces composée de 14 pistoles d'Espagne et 4 louis d'argent de 30 sols. L'inventaire après décès fit aussi mention de quelques petites rentes à prendre sur des particuliers ou sur la ville de Paris. Certaines de ces dernières pouvaient provenir de la succession de Geneviève de Chomedey, tante du défunt, qui en avait hérité pour un tiers comme sa sœur Jacqueline et sa cousine Catherine Alleaume, veuve de Marc-Antoine de Bresme, seigneur de Marchais. Un testament du 18 août 1644 déposé chez 
Grandrye, notaire au Châtelet, ${ }^{27}$ l'avait d'abord gratifié pour l'aider à payer ses dettes d'une somme de 200 livres renforcée d'un regret de ne pouvoir faire davantage. Puis, en exécution d'un acte de partage passé devant le même notaire après tirage au sort de billets mis dans le chapeau d'un petit garçon inconnu des parties, ${ }^{28}$ le 15 octobre 1647, il s'était vu attribuer 3 rentes de 103 livres, 6 sols, 8 deniers, 50 livres et 23 livres sur la ville de Paris. Maisonneuve avait, ensuite, par acte passé devant Chévignon, notaire à Troyes, le 10 août 1652, acquis la part de sa sœur Jacqueline, en échange de ses droits dans la succession de leur père. ${ }^{29}$

Paul de Chomedey mourut le 9 septembre 1676, le lendemain du jour où il avait dicté ses dernières volontés, dans sa chambre, au premier étage et vraisemblablement dans son lit. Lorsque Philippe de Turmenyes fit mettre les scellés par le commissaire au Châtelet François Poiret, le jour même, ${ }^{30}$ celui-ci vit le cadavre étendu sur une paillasse et cela pourrait donner à penser que le corps avait été mis sur celle du domestique, mais le lit personnel de Maisonneuve comportait, lui aussi, une paillasse. Il est un peu étonnant qu'elle ait attiré particulièrement l'attention du commissaire, car elle était recouverte d'un matelas revêtu de futaine, rempli de bourlanisse ou bourrelanisse, ${ }^{31}$ d'une couverture de laine blanche, d'un traversin rempli de plumes. Ce lit était à hauts piliers, garni de ferrure et entouré d'une tapisserie avec deux rideaux. Il s'agissait seulement d'une méchante tapisserie de bergame et l'expression «petite couche» employée lors de l'inventaire peut en définitive expliquer la quelque peu méprisante «paillasse » du commissaire qui qualifia de «méchant lit» celui qu'utilisait Louis Fin. Nous pouvons en tout cas penser avec certitude que le lit mortuaire était loin d'être somptueux.

27 Cf. Etude 5, no 98, à la date.

28 Cf. Idem, no 103.

$29 \mathrm{Cf}$. Inventaire après décès, déjà cité.

$30 \mathrm{Cf}$. Procès-verbal publié par Z. Massicotte, Bulletin des Recherches historiques de Québec, tome 22 (janvier-février 1916): $139,4^{\circ} \mathrm{Pa} 152$, à la Bib. Nat., d'après Y 15718, aux Archives Nationales.

31 On trouve le mot sous cette dernière forme dans les inventaires de l'époque: cf. Eudore Soulié, Recherches sur Molière et sa famille, pp. 222 et 250,1863, $8^{\circ} \operatorname{Ln}^{27} 14393$ à la Bib. Nat. Communication de Mme Jurgens, conservateur. 
Quelques autres obscurités des procès-verbaux sont plus ennuyeuses pour qui serait désireux d'entreprendre la critique d'un portrait reproduit par un de nos plus éminents prédécesseurs, ${ }^{32}$ donnant au gouverneur quelque peu l'apparence d'un chevalier du Moyen âge. Maisonneuve devait certainement posséder un manteau qui ne figura pas à l'inventaire. Étant donné qu'on y trouve des chemises, une omission des vêtements susceptibles d'être attribués à Louis Fin ne paraît pas vraisemblable. Il faut penser que Chomedey fut habillé après sa mort et que l'inventaire fut incomplet sans raison admissible, car le procès-verbal d'apposition des scellés signale un habit de droguet gris réclamé par Louis Fin qui ne figure pas à l'inventaire.

Le gouverneur possédait une quantité de linge de corps raisonnable avec 9 chemises dont 5 neuves et 5 caleçons dont 3 neufs et 2 vieux, ensemble estimé 15 livres. 9 mouchoirs, 5 paires de chaussettes, 3 coiffes de nuit et une douzaine de cravates correspondaient à peu près avec une garde-robe comprenant 2 justaucorps dont un de couleur minime, 2 hauts-de-chausse en culotte de droguet et une chemisette de ratine blanche. Ces vêtements devaient être passablement usagés, car ils furent estimés seulement 6 livres, bien qu'un des justaucorps eût été soigneusement recouvert de musc. Quatre vieux chapeaux de feutre noir, 4 paires de chaussons, 1 paire de bas d'estame couleur de minime, avec une perruque indispensable pour un officier du règne de Louis XIV permettent de reconstituer un ensemble complété par une épée avec son ceinturon qui ne semblent pas avoir été ensevelis avec le gouverneur.

Nous pouvons donc camper un Maisonneuve vêtu d'un vieux chapeau de feutre noir sur la perruque, d'un habit de droguet gris, c'est-à-dire d'une étoffe de laine de peu de valeur, d'un justaucorps marron, d'une culotte d'une couleur hélas ignorée, de bas de laine en tricot marron et de chaussons, l'aspect martial étant sauvegardé par l'épée et son ceinturon.

Pour en revenir au commissaire Poiret, celui-ci se trouva en présence de Philippe de Turmenyes, de Me François Le Chambre,

32 Cf. Le Jeune, Dictionnaire général du Canada (1932), inconnu à la Bib. Nat. 
prêtre de la paroisse St-Étienne du Mont, de Louis Fin, valet du défunt, d'Antoinette Hardelet, veuve de Charles Taconnet, sa garde-malade. Les scellés furent apposés seulement dans la chambre mortuaire sur la serrure d'un petit cabinet en planches de sapin, sur celle d'un grand coffre de bahut (sic) carré, couvert de cuir noir et sur un petit coffre de cuir que l'exécuteur testamentaire reconnut appartenir à Louis Fin. Il nota la comparution de la nièce et héritière Marie Bouvot, femme séparée de biens de Bernard de Baraillon, écuyer, sieur de Neuville, demeurant rue Mazarine, paroisse Saint-Sulpice. Assistée de son procureur, Louis de Villeneufve, elle déclara que le testament avait été suggéré. Turmenyes se tint sur la défensive, assisté, lui aussi, de son procureur nommé Périer.

L'inventaire fut effectué le surlendemain 11 septembre, par le notaire Bernard Mousnier ${ }^{33}$ et le sergent à verge, François Jacob, fit la prisée en présence de l'exécuteur testamentaire, de l'héritière, des deux procureurs et de Louis Fin, gardien des scellés. Ce dernier réclama 535 livres sept sols dont 264 en vertu de l'acte du 10 mai 1673,150 pour gages échus depuis cette date jusqu'au 10 mai 1676, précision indiquant qu'il gagnait 50 livres par an, soit environ $100000 \mathrm{fcs}$ par an, xaleur 1958, d'après une estimation fondée sur une solde d'officier, ${ }^{34} 16$ livres 10 sols pour gages depuis le 10 mai précédent, 34 livres, 10 livres et 61 livres, créances inscrites au dos de l'acte du 10 mai 1673. Cette sécurité prise en faveur de Louis Fin permet d'expliquer l'existence de ces dernières dettes pouvant sembler étranges, puisque Chomedey possédait 160 livres en argent comptant. On doit considérer d'abord que Louis Fin n'était pas dépensier, ensuite, qu'habitant avec son maître, une somme d'argent trouvée en sa possession lors du décès, aurait pu donner lieu à une contestation qui se trouvait évitée par l'attribution des espèces à la succession. On ne trouva dans la cave qu'une futaille en vidange, c'est-à-dire contenant une certaine quantité de vin, ensemble estimé 10 sols. Le mobilier de la petite salle basse était pauvre, comprenant une petite table, 6 petites chaises à dossiers de tapisserie, 2 douzaines

33 Cf. Etude 112, no 164.

34 Cf. Le livre de raison de François Tapie de Monteil, étude en préparation. 
d'images, 1 paire de chenêts, 1 paire de pincettes, 1 table en bois de sapin et 1 morceau de tapisserie.

Maisonneuve se tenait habituellement avec son domestique dans la chambre du premier étage où il mourut, car le mobilier comprenait le second lit destiné à Louis Fin, composé d'une paillasse, d'un matelas de bourlanisse, recouvert de toile peinte, sans doute par coquetterie, d'un petit oreiller recouvert de tapisserie, de deux couvertures, l'une en toile verte, l'autre faite de deux morceaux de tapisserie.

Les chenêts rompus ne valaient guère mieux que ceux de la salle basse, en dépit de leurs petites pommes de cuivre et c'était là que se faisait la cuisine à même la cheminée, comme le prouvent une crémaillère de fer, une broche avec son tourne-broche garni de poids de grès en poudre avec cordage, une poêle avec un trépied, une lèchefrite, un soufflet, les trois derniers objets valant vingt sols, enfin deux chaudrons de cuivre jaune garnis de leurs anses de fer.

L'éclairage était modestement assuré par un chandelier et deux petits flambeaux. La vaisselle était relativement importante avec 12 assiettes, 8 plats et 1 salière. On est un peu étonné de ne trouver qu'une seule tasse en étain, mais le commissaire Poiret avait constaté l'existence de 10 ou 12 livres d'étain. Par contre, 3 cuillers et 3 fourchettes d'argent, mieux décrites lors de l'inventaire comme étant façon de Paris, pesant 1 marc 3 onces, furent estimées 27 livres le marc, soit pour le lot, 37 livres 2 sols.

Maisonneuve aimait la musique, car il possédait un luth et ceci explique sans doute la disposition de son testament en faveur du maître Robert Carron.

Le luxe était représenté par deux miroirs, une vierge tenant un petit Jésus de terre cuite, façon marbre, environ 13 aunes de tapisserie faisant le tour de la pièce. D'un point de vue plus utilitaire, celle-ci n'était meublée, en plus des deux lits, que de 2 tables dont une brisée et du coffre carré recouvert de cuir noir avec serrure servant à clef et deux pieds de bois, meuble qui ne devait pas être fort beau, car on l'estima seulement 30 sols, soit 10 sols de plus que la poêle, la lèchefrite et le soufflet, environ 3000 fes 1958. 
Le linge de maison comprenait seulement 7 draps de toile de chanvre anciens et éliminés, 8 nappes, 8 serviettes de toiles différentes. Cette correspondance avec la vaisselle impliquerait l'éventualité d'invitations à partager les résultats des préparations culinaires limitées par le petit nombre des couverts d'argent mais aux possibilités renforcées par l'existence des étains.

Les travaux intellectuels étaient facilités par une petite écritoire en bois façon chagrin et une bibliothèque peu importante composée de livres pieux et d'ouvrages historiques généraux; l'inventaire signale les œuvres de $\mathrm{Mr}$ de Sales, c'est-à-dire de Saint François de Sales en 2 tomes reliés en veau, la Fleur des Saints en 2 tomes et le Catéchisme de Grenade. L'érudition n'apparaissait guère avec des Histoires romaine, d'Angleterre, des Flandres, de France en 3 tomes. Aucune indication n'est fournie au sujet de 30 autres volumes, mais nous aurions vraisemblablement été intéressé davantage par une liasse de pièces concernant les affaires du Canada, notamment celles des filles de la Congrégation de Notre-Dame-de-Montréal.

\section{II}

\section{Philippe de Turmenyes, mandataire des filles de la Congrégation de Notre-Dame-de-Montréal}

Philippe de Turmenyes fut gratifié par les filles de la Congrégation de Notre-Dame-de-Montréal d'une procuration générale consentie le 18 septembre 1677 devant Basset, notaire à Montréal, ${ }^{1}$ en exécution de laquelle et en utilisation du legs consenti par Maisonneuve, il versa, le 27 septembre 1677, à Gédéon du Metz, garde du Trésor royal, une somme de 2100 livres, prix d'acquisition d'une rente de 300 livres au denier 14, contrat régularisé le 18 janvier 1678.

Turmenyes demeurait rue Neuve, paroisse Saint-Médéric, le 18 avril 1679 , lorsqu'après avoir effectué le dépôt de sa procuration, le 15 mai 1678, en l'étude de Bernard Mousnier, il reçut, no 174 .

${ }^{1}$ Cf. Acte du 18 avril 1679, minutes de Bernard Mousnier, étude 112, 
en conséquence et comptant de Me Nicolas Raisin, avocat au parlement, demeurant rue Saint-Martin, paroisse Saint-Jacquesde-la-Boucherie, une somme de 35 livres. Il s'agissait d'une année d'arrérages de la rente constituée par l'avocat à sa sœur Marie, fille d'Edme Raisin et d'Anne Collot, à partir du 27 octobre 1677, jour du décès d'Edme Raisin. ${ }^{2}$

En vertu de la même procuration, il reçut, le 27 mai 1679, de Me François Le Mosny, prêtre, demeurant rue Pot-de-Fer, paroisse Saint-Sulpice, procureur des dames hospitalières de Montréal, ${ }^{3} 600$ livres en 54 louis d'or et pistoles pour le rachat d'une rente de 40 livres constituée par défunte Jeanne Mance, alors administratrice de l'hôpital de Montréal, au bénéfice des filles de la Congrégation suivant contrat passé devant Basset, notaire à Montréal, le 3 janvier 1676. Cette rente représentait un capital de 800 livres constitué au cours de la livre en NouvelleFrance subissant une dévaluation d'un quart sur le cours de la métropole.

Ce rachat fut accepté sous la condition d'être ratifié par les filles de la Congrégation qui s'exécutèrent, le 5 octobre 1679 , suivant acte passé devant Maugue, tabellion en l'île de Montréal, représentées par Marguerite Bourgeois, supérieure, Geneviève du Rozay, assistante, qui signa du Rozoy, Anne Hyou et Marguerite Sommillard avec l'agrément de messire Pierre Rémy, leur supérieur. Les religieuses hospitalières ratifièrent elles aussi par l'entremise de révérende mère Renée Le Jumeau, supérieure, de sœur Catherine Macé, assistante, de Marie Morin, hospitalière, de Catherine Denis, dépositaire administratrice du revenu temporel de l'hôpital. Marguerite Bourgeois remit l'acte elle-même au notaire parisien le $1^{\text {er }}$ février 1680 .

Les petites missions devant être accomplies pour le compte de la congrégation étaient diverses, car, suivant acte passé devant Mousnier, le 11 février 1681, ${ }^{4}$ Turmenyes remit à Jeanne Groisard, veuve de Zacharie Dupuy, écuyer, major de Montréal, une somme de 50 livres. Ce versement représentait un arrérage semes-

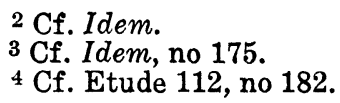


triel d'une rente annuelle de 100 livres consentie par Marguerite Bourgeois, supérieure de la congrégation, suivant acte passé avec l'assentiment de $\mathrm{M}^{\mathrm{gr}}$ de Laval, évêque de Québec, le 17 octobre 1680, devant Rageot et Duguet, notaires en cette ville. ${ }^{5}$ Il s'agissait d'une compensation au profit de la veuve Dupuy, obligée par sa santé, de quitter la maison des filles dont la congrégation conservait en contre-partie les biens donnés par le major de Montréal.

Le 13 février 1682, Turmenyes fit insinuer au Châtelet une fondation consentie devant Mousnier le 23 décembre 1681 par Catherine Douart, fille majeure, maîtresse lingère, demeurant rue du Four, à Saint-Germain-des-Prés. Il s'agissait d'une rente annuelle de 300 livres sur les prévôts des marchands de Paris, rachetable pour 6000 livres, destinée à fonder une place pour une fille sans dot que les religieuses hospitalières de l'hôpital SaintJoseph de Montréal choisiraient pour l'accepter parmi elles. Catherine Douart réservait l'usufruit de la rente pendant sa vie et celle de sa fille de boutique, Marie Joisan, mais la donation était perpétuelle, la rente devant être reportée dans les mêmes conditions au profit d'une autre religieuse après le décès de la bénéficiaire.

La brave lingère donna deux explications de sa générosité: le désir qu'elle avait éprouvé pendant plusieurs années de se faire religieuse sans parvenir à le réaliser et la prospérité apportée à son négoce par les prières des sœurs parmi lesquelles se trouvaient des parisiennes de sa connaissance. La donation fut acceptée d'abord par les demoiselles Marie-Madeleine Dorléans de Rothelin, fille majeure demeurant rue des Fossoyeurs, paroisse Saint-Sulpice, et Claude Colin, fille majeure demeurant rue Palais d'Orléans dans la même paroisse. Elles étaient pourvues d'une procuration des religieuses hospitalières donnée devant Maugue, notaire à Montréal, le 23 octobre 1680 et déposée en l'étude de Claude Le Vasseur, notaire au Châtelet, le 15 décembre 1681. Une ratification intervint le 22 octobre 1682 devant Maugue, notaire à Montréal, avec l'agrément de Gabriel Souart, geois.

5 Cf. Pièce annexée à l'acte précédent et signée de Marguerite Bour- 
curé de cette ville, par révérende mère Catherine Macé, sœur Judith de Moreau de Brézolles, sœur Renée Le Jumeau, sœur Charlotte Gallard et sœur Marie Morin. ${ }^{6}$

Marguerite Bourgeois, supérieure, Marie Barbier, assistante, Marguerite Gariepy, économe des filles de la congrégation de Notre-Dame-de-Montréal, gratifièrent Philippe de Turmenyes et Nicolas de Turmenyes, sieur de Chennevière, son neveu, demeurant à Paris, rue Neuve, paroisse Saint-Médéric, d'une procuration passée devant Maugue et Basset, notaires à Montréal, le dernier août 1693, et remise par Philippe de Turmenyes, demeurant rue Simon le Franc, paroisse de Saint-Médéric, le 10 septembre 1694, à Cáillet, notaire au Châtelet. ${ }^{\top}$ Cette procuration permit à l'oncle et au neveu demeurant tous deux rue Simon le Franc d'encaisser le 4 juin 1695, de Monseigneur de Saint-Vallier, ${ }^{8} 2,400$ livres en louis d'argent représentant le capital de la rente de 600 livres, monnaie de France, et de 800 livres, monnaie de Canada, dont il avait, suivant acte du 7 septembre 1693, passé devant Adhémar, notaire à Québec, gratifié les filles de la Congrégation. Celles-ci se trouvaient à la tête de 8400 livres, car les 6000 livres représentant leurs rentes précédentes avaient été remboursées suivant quittance du 26 mai 1695 donnée par Philippe de Turmenyes qui remploya l'ensemble en leur achetant 600 livres de rente au denier 14 sur les aides et gabelles par versement entre les mains de Jean Gruyn, garde du Trésor royal, le 20 juin suivant.?

Les filles de la Congrégation consentirent encore devant Maugue et Basset, notaires à Montréal, le $1^{\text {er }}$ novembre 1696, une procuration en faveur de Nicolas de Turmenyes alors qualifié sieur de Chevannes et Philippe de Turmenyes fit, le 25 mars 1698, à Jean de Turmenyes, garde du Trésor royal, le versement nécessaire pour reconstituer leur rente après un nouveau remboursement. ${ }^{10}$

Nous avons, malheureusement, perdu la trace de Philippe de

${ }^{6}$ Cf. Minutes de Bernard Mousnier, étude 112, no 186.

7 Cf. Etude 75, no 402.

8 Cf. Minutes de Lequin, étude 112, no 229.

9 Cf. Idem.

${ }^{10} \mathrm{Cf}$. Idem, no 234. 
Turmenyes qui mourut célibataire, le 3 août 1699, à l'âge de 69 ans. ${ }^{11}$

ROBERT LE BLANT

24 rue Varize, Paris XVI

\section{Pièces justificatives}

Monsieur de Maison neufve, J'ai sceu par les relations qui m'ont esté envoyées de la Nouvelle France, l'estat des lieux et le grand péril ou se trouvent les habitans de l'Isle de Montréal par les incursions des hyroquois ennemis de la foy et le pressant besoing qu'ils ont de secours pour se preserver de leur violence. A quoy desirant apporter le reméde convenable et seconder le zèle des personnes de piété qui travaillent par dela a la conversion des sauvages, j'ay eu bien agréable le secours d'hommes qu'ilz ont accueilly par leurs soings pour la deffense de l'Isle et particuliérement du fort auquel ils vous ont estably et vous ordonne de travailler incessamment pour les réunir ensemble et les conduire soubs mon authorité en laditte Isle de Montréal par les vaisseaux que vous trouverez plus commodes dans mes ports avec les provisions, esquipages, armes et munitions nécessaires à leur deffense et y faire vivre les habitans en paix et procurer esdits lieux autant qu'il vous sera possible l'estendue de la foy et l'establissement des colonnies francoises

Sur ce je prie dieu qu'il vous ayt Monsieur de Maison Neufve en sa sainte garde.

Ecrit à Paris le dernier jour, d'avril 1653.

Louis-De Loménie.

Au dos: A Monsieur de Maison neufve gouverneur et commandant dans le fort de Montréal en la Nouvelle France. ${ }^{12}$

II

Aujourd'huy est comparu par devant les notaires et gardenottes du roy au Chatelet de Paris soubzsignez Paul de Chomedey

11 Cf. Dossiers Bleus 650, art. Turmenyes, déjà cité.

12 Signatures autographes et cachet à 3 fleurs de lys. Pièce annexée au certificat du 22 novembre 1670, Minutier Central, minutes de Mousnier, étude 112 , no 134. 
escuyer, sieur de Maisonneusve cy devant gouverneur de l'Isle de Montreal en la Nouvelle France estant de present à Paris logé sur les fossez d'entre les portes de St Marcel et de St Victor

Lequel a déclaré qu'en l'année 1653 il auroit receu ordre de sa maiesté par une lettre de cachet du 8 avril de ladite année de conduire en ladite Isle un secours considérable pour la deffendre contre les incursions des Yroquois ennemys de la foy

A ce subiet, Marguerite Bourgeois, fille natifve de la ville de Troyes en Champagne, demeurant en icelle rue du Chaudron aagée pour lors de trente trois ans, désireuse de contribuer de tout son possible à l'establissement de la foy dans ledit pays de la Nouvelle France et particulierement pour l'instruction des jeunes enfants dudit pays seroit allé trouver ledit sieur de Maisonneuve à Nantes en Bretagne, lieu de l'assemblée dudit secours et se seroit embarquée dans le mesme navire ou estoit ledit sieur de Maisonneufve pour passer en la Nouvelle France, ce qu'elle auroit fait en randant pendant le voyage tous les services de charité possibles à tous ceux qui estoient dans le navire;

que laditte Bourgeois estant arrivée en ladite Isle de Montréal se seroit employée avec tout le zele possible, non seulement à l'instruction de la jeunesse mais de plus à touttes les œuvres de charité et de piété qui se sont rencontrées en touttes sortes d'occasions pour le bien public en considération desquelles choses cy dessus et pour luy donner moyen de continuer dans ces mesmes employs, il luy auroit donné diverses concessions de terres, lesquelles ladite Bourgeois auroit fait desfricher à ses despens et fait bastir une métairie et l'auroit fournie des bestiaux nécessaires et de plus auroit a ses mesmes despens fait bastir deux corps de logis pour le logement des filles proche le port et hospital St Joseph de Villemarie en ladite Isle de Montreal; que pour travailler à l'augmentation dudit establissement laditte Bourgeois auroit fait un voyage en France en l'annee 1658 ou elle se seroit associé trois filles avec lesquelles elle seroit retournée dans ledit pays pour y continuer les mesmes fonctions qu'elle auroit executé avec zèle et charité jusques en la presente année qu'il a esté jugé à propos pour le bien dudit pays que ladite Bourgeois passast en France pour faire en sorte d'avoir augmantation de filles pour sa communauté et pour faire en sorte d'avoir des lettres patentes de Sa Majesté pour son dernier establissement; que ladite Bourgeois estant arrivée en cette ville pour ce subjet au mois d'octobre dernier, luy auroit communiqué les certificats qu'elle auroit pour certifier de Monsieur l'Evesque de Petrée, de Monsieur de Courcelles, lieutenant-général de $\mathrm{Sa}$ Majesté audit pays et de Monsieur Talon, Intendant pour saditte majesté au mesme pays avec le 
résultat de l'assemblée des habitans de ladite Isle de Montreal et le certificat du curé du mesme lieu de Montreal et est ladite lettre de cachet devant mentionnée jointe à la presente minutte pour y avoir recours et en estre deslivré des expéditions a qui il appartiendra.

Ledit sieur de Maisonneuve faisant les declarations cy dessus pour servir a ladite Bourgeois pour sondit establissement ainsy qu'elle le jugera plus à propos pour le bien d'iceluy dont et de quoy ledit sieur de Maisonneufve a requis acte a luy octroyé le present en l'estude de Mousnier l'un desdits notaires soubzignés le vingt deux ${ }^{\mathrm{e}}$ jour de novembre mil sex cens soixante dix apres midy et a signé

Paul de Chomedey, Auvray, Mousnier ${ }^{13}$

\section{III}

Fut présent Paul Chomedey, escuyer, sieur de Maisonneufve cy devant gouverneur de l'Isle de Montreal en la Nouvelle France demeurant à Paris sur le fossé d'entre les portes St Marcel et St Victor paroisse St Estienne du Mont gisant au lit malade de corps en une antresolle au premier estage ayant veue sur la cour des R.P. de la doctrine chrestienne et sur le fossé, sain touteffois d'esprit, memoire et entendement comme il nous est apparu par ses parolles gestes et maintien lequel considérant la certitude de la mort et qu'il n'y a rien de sy incertain que l'heure et le moment d'icelle et craignant d'en estre prèvenu avant qu'avoir disposé de ses dernières volontez a fait son testament et ordonnance de derniere volonté qu'il nous a dicté et nommé ansy qu'il ensuict Premiérement comme chrestien catholique romain a recommandé son ame a Dieu supliant sa divine bonté de luy faire misericorde et la sainte Vierge, saints et saintes d'intercédes pour luy, ordonné l'inhumation de son corps en l'église desdits R.P. de la Doctrine chrestienne de la maison de St Charles ausquels il donne et lègue tant pour le droit de sepulture que pour les priéres qu'ils luy feront faire à leur discretion la somme de cinq cens livres une fois payée se raportant ledit sieur testateur au surplus de ses obséques services et priéres à la discretion et dévotion de son executeur testamentaire soubs nommé

Item donne et lègue à la congregation Notre Dame de Montreal en la Nouvelle France dont Marguerite Bourgeois est superieure la somme de deux mille livres une fois payée

13 Minutier Central, minutes de Mousnier, étude 112, no 134. 
Donne et lègue à Louis Fin son serviteur domestique la somme de trois cens livres une fois payée sans diminution de ce qu'il luy doibt tant par obligation que pour ses gages escheuz depuis ladite obligation

Item donne et lègue aux hospitalliéres de S Joseph de Villemarie dans ladite Isle de Montreal la somme de mil livres une fois payée

Item aux filles de la Congregation de la ville de Troyes ou ses soeur et niepce sont religieuses la somme de mille livres aussy une fois payée

A Monsieur Le Bey promoteur de Monseigneur l'evesque de Troyes la somme de trois cens livres

Item, aux pauvres de l'hopital de Sainte-Reyne la somme de quatre cens livres le tout une fois payée

Déclare ledit testateur qu'il doibt à Jacques Le Bert et Charles Le Moyne marchands en Canada l'environ quatre cens livres sur les comptes qu'ilz lui ont envoyez et qui se trouveront dans ses papiers

Plus qu'il doibt mil livres de principal faisant rente a la damoiselle de Saint Jacques

Toutes lesquelles debtes et legs cy dessus faitz et declarez ledit sieur testateur veult estre pris sur les dix mil huit cens livres principal de six cens livres de rente au denier dix huit que lui doibt la communauté des marchands de la ville de Troyes et arrerages qui s'en trouveront deubz

Item donne et lègue a Messire Philippes de Turmenyes bourgeois de Paris son bon amy tous les meubles meublans, hardes, linge, habit, vaisselle et autres ustancilles de Mesnage et autres qui se trouveront au jour de son deceds ez lieu ou il demeure presentement ou autre ou il pourra demeurer au jour de son deceds sans aucune réserve sy ce n'est de quelques chemises et habit qui puisse estre à l'usage dudit Fin qu'il prie ledit de Turmenyes luy laisser

Et outre donne et lègue audit sieur de Turmenyes moitié de ce qui se trouvera rester du principal et arrerages de ladite rente apréslesdites debtes et legs pris sur iceux; et quand à l'autre moitié il veut qu'elle soit mise entre les mains du R.P. Charles provincial desdits R.P. de la Doctrine chrestienne cy dessus pour en disposer par lui pieusement comme il advisera, voulant touteffois que sur le total dudit restant il soit pris la somme de deux cens livres une fois payée qu'il donne et lègue au sieur Robert Carron Me joueur de luth et pour executer le présent testament 
ledit sieur testateur nomme et eslit la personne dudit sieur de Turmenyes le priant d'en prendre la peyne, se dessaississant en ses mains de tous ses biens a cet effect, revocquant tous autres testaments et codicilles qu'il pourroit avoir faict avant le present auquel il s'arrête comme estant sa dernière volonté.

Ce fut ainsi fait, dicté et nomé par ledit sieur testateur a nous notaires soubssignez puys à luy par l'un des notaires, l'autre present releu qu'il a dit bien scavoir et entendre et a persévéré en ladite entresolle l'an mil six cens soixante seize le huictiesme de septembre neuf heures du soir et a signé

Paul de Chomedey Torinon Aumont. ${ }^{14}$

14 Minutier Central, minutes d'Aumont, Etude XVII, septembre à décembre 1676, no 364 . 